\title{
Multivalley spin relaxation in $n$-type bulk GaAs in the presence of high electric fields
}

\author{
H. Tong and M. W. Wu* \\ Hefei National Laboratory for Physical Sciences at Microscale and Department of Physics, \\ University of Science and Technology of China, Hefei, Anhui, 230026, China
}

(Dated: November 6, 2018)

\begin{abstract}
Multivalley spin relaxation in $n$-type bulk GaAs in the presence of high electric field is investigated from the microscopic kinetic spin Bloch equation approach with the $\Gamma$ and $L$ valleys included. We show that the spin relaxation time decreases monotonically with the electric field, which differs from the two-dimensional case and is recognized due to to the cubic form of the Dresselhauss spin-orbit coupling of the $\Gamma$ valley in bulk. In addition to the direct modulation of the spin relaxation time, the electric field also strongly influences the density and temperature dependences of the spin relaxation. In contrast to the monotonic decrease with increasing lattice temperature in the field-free condition, the spin relaxation time is shown to decrease more slowly under the influence of the electric field and even to increase monotonically in the case with small electron density and high electric field. We even predict a peak in weakly doped samples under moderate electric field due to the anomalous lattice-temperature dependence of the hot-electron temperature. As for the $L$ valleys, we show that instead of playing the role of a "drain" of the total spin polarization as in quantum well systems, in bulk they serve as a "momentum damping area", which prevents electrons from drifting to higher momentum states. This tends to suppress the inhomogeneous broadening and hence leads to an increase of the spin relaxation time.
\end{abstract}

PACS numbers: 72.25.Rb, 72.20.Ht, 71.10.-w, 71.70.Ej

\section{INTRODUCTION}

Semiconductor spintronics, which aims at incorporating the spin degree of freedom in electronics, has attracted much attention in the past decades $\underline{\underline{1}} \underline{\underline{8}}$ One of the main challenges in realizing spintronic devices lies in the control of spin lifetime. Many investigations have been devoted in bulk III-V semiconductors $\underline{\underline{9}} \underline{\underline{30}}$ Various factors on spin relaxation, such as carrier and impurity densities,,$\underline{-20}$ temperature,,$\underline{18-25}$ mobility,, 23 initial spin polarization ${ }^{20}$ and electric $\underline{20,28}$ and magnetic fields, $\stackrel{10}{\underline{12}, 17,24}$ etc, have been extensively studied. Remarkably, spin relaxation time (SRT) as long as $130 \mathrm{~ns}$ is observed in $n$-type GaAs $s^{10,11}$ and the SRT varying by more than three orders of magnitude with temperature or density is reported $\underline{\underline{10}}$ Very recently, Jiang and $\mathrm{Wu}$ performed a systematic investigation on the electron spin relaxation in bulk III-V semiconductors in metallic regime from the fully microscopic kinetic spin Bloch equation (KSBE) approach ${ }^{20}$ The D'yakonovPerel' (DP) mechanism 31 is demonstrated to be dominant in $n$-type III-V semiconductors, even in narrow band ones, and many features in contrast to the previous understandings in the literature are reported. $\stackrel{20}{ }$ Some of their predictions have been soon realized in the subsequent experimental investigations $\underline{12}-16,24-27$ Moreover, the very recent works 29,30 on the spin relaxation near the metal-to-insulator transition have extended the investigation in that regime back in $2002, \underline{11}$

It is noted that works discussed above mainly concern electron spin relaxation in the absence of or with relatively low electric fields. In Ref. 20, an electric field applied up to $2 \mathrm{kV} / \mathrm{cm}$ is also considered and the SRT is found to be effectively manipulated, especially for the low temperature case, where the SRT is suppressed down to $1 / 10$ of its original value with a small electric field up to $0.05 \mathrm{kV} / \mathrm{cm}$. Clear hot-electron effect is shown and the different field dependence of the SRT compared to that in quantum wells is addressed. Nevertheless, since most current electronic devices are performed in the highfield conditions, new features of the SRT are expected when electrons are drifted to high valleys. ${ }^{32}$ The different spin-orbit couplings (SOCs), momentum relaxations and effective masses in different valleys should have pronounced effects on spin dynamics. In fact, investigations on how the spin relaxation is affected by high electric field have been carried out in quantum well systems. The hot-electron effect $\frac{33}{3}$ and the multi-subband effect $\frac{34}{4}$ on spin dephasing in $n$-type GaAs quantum wells have been studied. Different field dependences of SRT are observed for different temperatures, well widths and initial spin polarizations. Moreover, the multivalley spin relaxation under high in-plane electric fields has been investigated in $n$-type GaAs quantum wells by taking into account the $\Gamma$ and $L$ valleys $\frac{35}{3}$ It is predicted that although the SOC in the $L$ valleys is much larger than that of the $\Gamma$ valley, 36 the spin polarization in the $L$ valleys shares the same damping rate as that in the $\Gamma$ valley. The $L$ valleys are pointed out to play the role of a "drain" of the total spin polarization due to the large SOC therein. Nonmonotonic dependence of the SRT on electric field has been reported, while the spin Gunn effect,,$\frac{37}{2}$ the spontaneous spin-polarization generation in the high-electricfield charge Gunn region, is pointed out to hardly realize in GaAs quantum wells. Despite these works in two-dimensional structures, a detailed fully microscopic study in bulk system under high electric field is still ab- 
sent. How the temperature and electron density dependences of the SRT are affected by the high electric field is still unclear and the feasibility of the spin Gunn effect predicted in bulk ${ }^{37}$ needs to be checked. Because of the cubic form of the Dresselhauss SOC in the $\Gamma$ valley as well as the absence of quantum confinement in bulk, different behaviors in spin relaxation are expected.

In this work, we apply the KSBEs to investigate the electron spin relaxation in $n$-type bulk GaAs in the presence of high electric fields with the $\Gamma$ and $L$ valleys included. The electric field dependence of the SRT is calculated and found to be monotonic, in contrast with the results in quantum wells. 35 We attribute this to the strong enhancement of inhomogeneous broadening $7,38,39$ resulting from the cubic form of the $\Gamma$-valley Dresselhaus SOC in bulk. The electric field is also shown to effectively change the density and the temperature dependences of the SRT. Remarkably, a peak is predicted in the temperature dependence of the SRT for relative low electron density under a moderate electric field. The underlying physics is analyzed. As for the $L$ valleys, we show that in contrast to the two-dimensional system where the $L$ valleys play the role of a "drain" of the total spin polarization, unexpectedly in bulk their effect on the total spin dynamics is weak and the total spin relaxation is mainly determined by the $\Gamma$ valley. It is shown that even with much larger SOC in the $L$ valleys, the inclusion of the $L$ valleys results in a longer rather than a shorter SRT. We find that the $L$ valleys serve as a "momentum damping area" where electrons are hardly to drift to higher momentum states due their large effective mass. This tends to suppress the inhomogeneous broadening and hence leads to the increase of the SRT.

This paper is organized as follows: In Sec. II, we set up our model and construct the KSBEs. In Sec. III, we lay out our main numerical results. The effects of electric fields, together with the carrier density, temperature and intervalley scattering (note that in this paper, with the term "intervalley scattering", we always indicate the $\Gamma-L$ intervalley scattering unless otherwise specified) on the SRT are discussed. We summarize in Sec. IV.

\section{MODEL AND KSBES}

We start our investigation in $n$-type bulk GaAs where the four $L$ valleys locate at $L$ points $\left[\mathbf{K}_{L_{i}}^{0}=\frac{\pi}{a_{0}}(1, \pm 1, \pm 1)\right.$ with $a_{0}$ denoting the lattice constant and $\left.i=1-4\right]$ and lie energetically $E_{\Gamma L}=0.296 \mathrm{eV}$ above the $\Gamma$ valley ${ }^{40} \mathrm{It}$ is noted that the four $L$ valleys can be arbitrarily chosen from the eight $L$ points limited by the condition that there is no center inversion symmetry between any two of them. In the spherically symmetric approximation, the electron effective masses of the $\Gamma$ and $L$ valleys are represented as $m_{\Gamma}=0.067 m_{0}$ and $m_{L}=0.23 m_{0},{ }^{41,42}$ respectively, with $m_{0}$ representing the free electron mass. We do not consider valleys of higher energy, e.g., the next-nearest valleys $X$ which are $E_{\mathrm{LX}}=0.166 \mathrm{eV}$ above the $L$ valleys, since even for the highest electric field $(E=8 \mathrm{kV} / \mathrm{cm})$ employed in this work, the fractions of electrons in these valleys are negligible. 32

The KSBEs derived from the nonequilibrium Green function method reads $7,35,38,39$

$$
\partial_{t} \rho_{\lambda \mathbf{k}_{\lambda}}=\left.\partial_{t} \rho_{\lambda \mathbf{k}_{\lambda}}\right|_{\text {coh }}+\left.\partial_{t} \rho_{\lambda \mathbf{k}_{\lambda}}\right|_{\text {drift }}+\left.\partial_{t} \rho_{\lambda \mathbf{k}_{\lambda}}\right|_{\text {scat }},
$$

in which $\rho_{\lambda \mathbf{k}_{\lambda}}$ is the density matrix of electrons with momentum $\mathbf{k}_{\lambda}$ in $\lambda$ valley. Note that $\mathbf{k}_{\lambda}$ is defined in reference to the valley center in each valley. The diagonal term $\rho_{\lambda \mathbf{k}_{\lambda}, \sigma \sigma} \equiv f_{\lambda \mathbf{k}_{\lambda}, \sigma}(\sigma= \pm 1 / 2)$ describes the distribution of each spin band and the off-diagonal term $\rho_{\lambda \mathbf{k}_{\lambda}, \frac{1}{2}-\frac{1}{2}}=\rho_{\lambda \mathbf{k}_{\lambda},-\frac{1}{2} \frac{1}{2}}^{*}$ is the correlation between the two spin bands. The coherent term is given by

$$
\left.\partial_{t} \rho_{\lambda \mathbf{k}_{\lambda}}\right|_{\mathrm{coh}}=-i\left[\boldsymbol{\Omega}_{\lambda}\left(\mathbf{k}_{\lambda}\right) \cdot \boldsymbol{\sigma}+\Sigma_{\mathrm{HF}}^{\lambda}\left(\mathbf{k}_{\lambda}\right), \rho_{\lambda \mathbf{k}_{\lambda}}\right],
$$

where [, ] is the commutator and $\boldsymbol{\Omega}_{\lambda}\left(\mathbf{k}_{\lambda}\right)$ represents the Dresselhaus SOC in $\lambda$ valley. $\frac{43}{}$ By setting the [100] and [010] directions as $x$ - and $y$-axises, respectively, $\boldsymbol{\Omega}_{\Gamma}\left(\mathbf{k}_{\Gamma}\right)$ takes the form 43,44

$$
\begin{aligned}
\mathbf{\Omega}_{\Gamma}\left(\mathbf{k}_{\Gamma}\right)= & \gamma_{D}\left[k_{\Gamma x}\left(k_{\Gamma y}^{2}-k_{\Gamma z}^{2}\right), k_{\Gamma y}\left(k_{\Gamma z}^{2}-k_{\Gamma x}^{2}\right),\right. \\
& \left.k_{\Gamma z}\left(k_{\Gamma x}^{2}-k_{\Gamma y}^{2}\right)\right] .
\end{aligned}
$$

By further denoting $\hat{\mathbf{n}}_{1 / 3}=(1,1, \pm 1) / \sqrt{3}$ and $\hat{\mathbf{n}}_{2 / 4}=$ $-(1, \pm 1,1) / \sqrt{3}$, which are the unit vectors of the longitudinal principle axis of the $L_{i}$ valleys, we have for the $L$ valleys $35,43,44$

$$
\boldsymbol{\Omega}_{L_{i}}\left(\mathbf{k}_{L_{i}}\right)=\beta_{D}\left(k_{L_{i x}}, k_{L_{i} y}, k_{L_{i} z}\right) \times \hat{\mathbf{n}}_{i} .
$$

$\Sigma_{\mathrm{HF}}^{\lambda}\left(\mathbf{k}_{\lambda}\right)=-\sum_{\mathbf{k}_{\lambda}^{\prime}} V_{\lambda \lambda, \mathbf{k}_{\lambda}-\mathbf{k}_{\lambda}^{\prime}} \rho_{\lambda \mathbf{k}_{\lambda}^{\prime}}$ in Eq. (2) is the Coulomb Hatree-Fock term with $V_{\lambda \lambda, \mathbf{k}_{\lambda}-\mathbf{k}_{\lambda}^{\prime}}$ representing the intravalley Coulomb scattering matrix element. The drift term takes the form $\left.\partial_{t} \rho_{\lambda \mathbf{k}_{\lambda}}\right|_{\text {drift }}=e \mathbf{E} \cdot \nabla_{\mathbf{k}_{\lambda}} \rho_{\lambda \mathbf{k}_{\lambda}}$ $(e>0) .\left.\quad \partial_{t} \rho_{\lambda \mathbf{k}_{\lambda}}\right|_{\text {scat }}$ stands for the scattering terms, which include intra- and inter-valley parts with the explicit expressions given in Appendix A.

\section{NUMERICAL RESULTS}

In this section, we present our results obtained by numerically solving the KSBEs following the scheme laid out in Refs. 20 and 35 . All parameters used in our computation are listed in Table I.

The initial spin polarized state of the system is prepared in the similar way as in Ref. 35, starting from the unpolarized equilibrium state. That state is first driven to the drifted steady state under the electric field. The main difference lies that after driving the system to the steady state, we turn on a circularly polarized laser pulse at $t_{1}=6$ ps to excite spin polarized electrons with a degree of injected spin polarization $P_{\text {inject }}=50 \%$ into the $\Gamma$ valley: ${ }^{1,45} \delta f_{\Gamma \mathbf{k}_{\Gamma}, \sigma}=\alpha_{\sigma} \exp \left[-\left(\varepsilon_{\mathbf{k}_{\Gamma}}^{\Gamma}-\varepsilon_{\text {pump }}\right)^{2} / 2 \delta_{\varepsilon}^{2}\right][1-$ $\left.f_{\Gamma \mathbf{k}_{\Gamma}, \sigma}\left(t_{1}\right)\right]$. Here $\alpha_{\sigma}=n_{\mathrm{pump}, \sigma} /\left\{\sum_{\mathbf{k}_{\Gamma}} \exp \left[-\left(\varepsilon_{\mathbf{k}_{\Gamma}}^{\Gamma}-\right.\right.\right.$ 
$\left.\left.\left.\varepsilon_{\text {pump }}\right)^{2} / 2 \delta_{\varepsilon}^{2}\right]\left[1-f_{\Gamma \mathbf{k}_{\Gamma}, \sigma}\left(t_{1}\right)\right]\right\} . \varepsilon_{\text {pump }}$ is the energy of pulse center in reference to the band minimum and $\delta_{\varepsilon}=\hbar / \delta_{\tau}$ with $\delta_{\tau}$ denoting the pulse width. $n_{\mathrm{pump}, \sigma}$ is the density of electrons with spin $\sigma$ after excitation with $n_{\text {pump }, \frac{1}{2}}=$ $3 n_{\text {pump },-\frac{1}{2}}$. In this work, we employ $\varepsilon_{\text {pump }}=4 \mathrm{meV}$ for the case of resonant excitation, $\delta_{\tau}=0.01 \mathrm{ps}$ and $n_{\text {pump }}=n_{\text {pump }, \frac{1}{2}}+n_{\text {pump },-\frac{1}{2}}=0.02 \times n_{e}$. Here $n_{e}$ stands for the unpolarized electron density before pumping which is equal to the doping density, so the total spin polarization after the pump pulse is $P_{0} \approx 1 \%$. It is noted that due to the strong electron-electron Coulomb scattering, the drifted steady-state distribution is established within $0.1 \mathrm{ps}$ after the pump pulse. Therefore the pulse characters, i.e., $\varepsilon_{\text {pump }}$ and $\delta_{\tau}$, have little influence on the SRT. It is further noted that due to the small initial spin polarization, the exact value of the polarization has marginal effect on the SRT $\underline{20}$

In Fig. 1, we plot the typical time evolution of the spin polarization and also the electron population in $\lambda$ valley in the condition with electric field $E=6 \mathrm{kV} / \mathrm{cm}$, electron density $n_{e}=10^{16} \mathrm{~cm}^{-3}$ and temperature $T=300 \mathrm{~K}$. Clear transfer of electrons from the $\Gamma$ valley to the $L$ valleys is observed. In order to quantitatively understand the influence of the $L$ valleys, we calculate the steadystate drift velocities of $\lambda$-valley electrons, the mobilities, the electron populations and the hot-electron temperatures in the $\Gamma$ and $L$ valleys as function of electric field. The explicit results are plotted in Appendix B with underlying physics analyzed. From Fig. 6(a) of Appendix B, one finds that our model is validated against the experimental data. 32

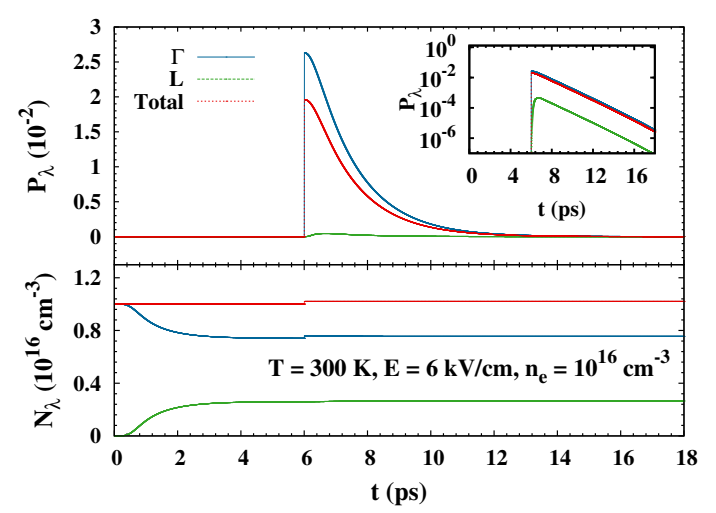

FIG. 1: (Color online) Typical evolution of the electron spin polarization and population in $\lambda$ valley in the condition with $n_{e}=10^{16} \mathrm{~cm}^{-3}, E=6 \mathrm{kV} / \mathrm{cm}$ and $T=300 \mathrm{~K}$.

In the following, we focus on how the SRT is influenced by the electric field, carrier density and lattice temperature. The effect of the $L$ valleys is also studied. The SRT $\tau$ is obtained by fitting temporal evolution of spin polarization $P_{\lambda}$ with an exponential decay (see the inset of Fig. (1). Throughout this work, the electric field is applied in the $x$-direction and its strength is limited up to
$8 \mathrm{kV} / \mathrm{cm}$ where the $X$ valleys can be neglected $3^{32}$

TABLE I: Material parameters used in the calculation (from Refs. 35 and 40 unless otherwise specified).

\begin{tabular}{llll}
\hline \hline$E_{g}^{\Gamma}(\mathrm{eV})$ & 1.519 & $\Omega_{\Gamma \Gamma}(\mathrm{meV})$ & 35.4 \\
$E_{g}^{L}(\mathrm{eV})$ & 1.815 & $\Omega_{\mathrm{L}_{\mathrm{i}} \mathrm{L}_{\mathrm{i}}}(\mathrm{meV})$ & 34.3 \\
$E_{g}^{X}(\mathrm{eV})$ & 1.981 & $\Omega_{\Gamma \mathrm{L}}(\mathrm{meV})$ & 20.8 \\
$m_{\Gamma}^{*} / m_{0}$ & 0.067 & $\Omega_{\mathrm{L}_{\mathrm{i}} \mathrm{L}_{\mathrm{j}}}(\mathrm{meV})$ & 29.0 \\
$m_{L}^{*} / m_{0}$ & 0.23 & $D_{\mathrm{L}_{\mathrm{i}} \mathrm{L}_{\mathrm{i}}}\left(10^{9} \mathrm{eV} / \mathrm{cm}\right)$ & 0.3 \\
$\kappa_{0}$ & 12.9 & $D_{\Gamma \mathrm{L}}\left(10^{9} \mathrm{eV} / \mathrm{cm}\right)$ & 1.1 \\
$\kappa_{\infty}$ & 10.8 & $D_{\mathrm{L}_{\mathrm{i}} \mathrm{L}_{\mathrm{j}}}\left(10^{9} \mathrm{eV} / \mathrm{cm}\right)$ & 1.0 \\
$\gamma_{\mathrm{D}}\left(\mathrm{eV} \cdot \AA^{3}\right)$ & $23.9^{a}$ & $d\left(10^{3} \mathrm{~kg} / \mathrm{m}^{3}\right)$ & 5.36 \\
$\beta_{\mathrm{D}}(\mathrm{eV} \cdot \AA)$ & 0.26 & & \\
\hline \hline
\end{tabular}

\section{A. Electric field dependence and effect of $L$ valleys}

We first study the electric field dependence of the SRT at $T=300 \mathrm{~K}$. In Fig. 22 we plot the SRTs against electric field for electron densities $n_{e}=10^{16}, 10^{17}$ and $10^{18} \mathrm{~cm}^{-3}$, corresponding to the nondegenerate, intermediate and degenerate regimes in the field-free condition, respectively. It is seen that the SRT is effectively modulated by the electric field. For all three cases, the SRTs decrease monotonically with the electric field and reach down to the ones with one order of magnitude smaller than the corresponding field-free values at $E=8 \mathrm{kV} / \mathrm{cm}$. This is different from the previous work in $n$-type GaAs quantum wells where a nonmonotonic electric field dependence is observed, $\stackrel{33-35}{35}$ but consistent with the very recent work in bulk GaAs by Jiang and $\mathrm{Wu}$ where the electric field is applied up to $2 \mathrm{kV} / \mathrm{cm} .20$ In the regime with $E<2 \mathrm{kV} / \mathrm{cm}$, according to Fig. 6)(c) in Appendix B, the $L$ valleys are still irrelevant. Therefore the underlying physics of the monotonic decrease of the SRT is the same as that analyzed in Ref. 20: Due to the cubic form of the Dresselhauss SOC in bulk, the enhancement of inhomogeneous broadening from the drift effect and the hot-electron effect [see Fig. 6(d) in Appendix B where the two-hot-electron-temperature behavior of $\Gamma$-valley electrons is discussed] is more pronounced than that in quantum wells with small well width where the SOC is in the linear form. $\underline{3-35}$ It hence overtakes the enhancement of momentum scattering and leads to the monotonic field dependence of the SRT. However, when the electric field is increased over $2 \mathrm{kV} / \mathrm{cm}$, a visible amount of electrons are drifted into the $L$ valleys [see Fig.6(c) in Appendix B] and hence the $L$ valleys are expected to play a role in the total spin relaxation.

An important feature of the multivalley spin relaxation can be noticed in the inset of Fig. 1 that the evolutions of spin polarizations in the $\Gamma$ and $L$ valleys share the same damping rate. This can be understood in respect to the rapid exchange of electrons between the $\Gamma$ and $L$ valleys resulting from the strong intervalley scattering. As shown in Fig. 3(a), by removing the intervalley 


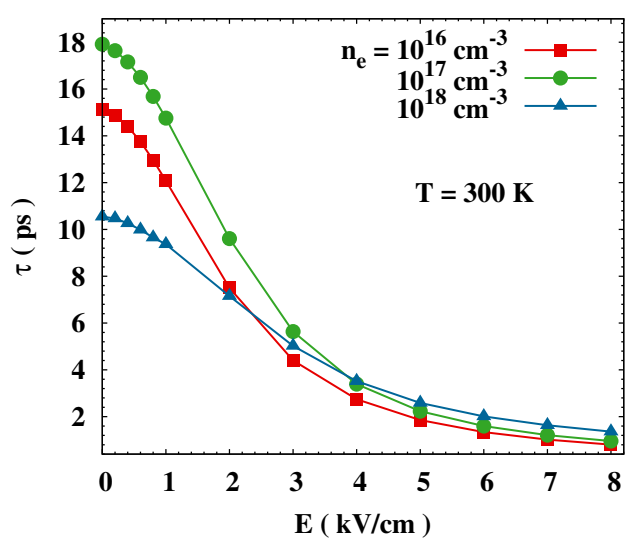

FIG. 2: (Color online) SRT $\tau$ vs. electric field $E$ at temperature $T=300 \mathrm{~K}$ for three electron densities: $n_{e}=10^{16}, 10^{17}$ and $10^{18} \mathrm{~cm}^{-3}$.

scattering $H_{\Gamma L}$, one finds that the spin polarizations in separate valleys now evolve independently and the "intrinsic" SRT in the $L$ valleys (curves with $\diamond$ ) becomes one order of magnitude smaller than that of the $\Gamma$ valley (curve with $\square$ ). This demonstrates the crucial role of the intervalley scattering in obtaining the identical damping of spin polarizations in the $\Gamma$ and $L$ valleys. To be more specific, we also calculate the SRT in each valley by removing only the intervalley electron-phonon or electron-electron Coulomb scattering. It is seen from Fig. 3(a) that the SRTs in the $\Gamma$ and $L$ valleys without the intervalley electron-electron Coulomb scattering coincide, whereas those without the intervalley electronphonon scattering are far away from each other. This demonstrates that the identical damping of spin polarizations in the $\Gamma$ and $L$ valleys comes from the intervalley electron-phonon scattering. It is noted that the fast spin relaxation in the $L$ valleys hints that they may serve as a "drain" of the total spin polarization, just as the case in quantum wells $\frac{35}{2}$

To further elucidate the role of the $L$ valleys in the bulk system, we calculate the SRTs by artificially removing the $L$ valleys [labeled as Case (ii)] and by setting the SOC in the $L$ valleys to zero [labeled as Case (iii)] in the computation. The calculated SRTs are compared to those obtained in the genuine condition [labeled as Case (i)]. In Fig. 3(b), we plot the SRTs as function of electric field for Case (i)-(iii) with $n_{e}=10^{18} \mathrm{~cm}^{-3}$ and $T=300 \mathrm{~K}$. One observes that for $E<2 \mathrm{kV} / \mathrm{cm}$, the effect of the $L$ valleys is marginal due to little electrons drifted into the $L$ valleys under weak electric field. When the electric field further increases, the $L$ valleys start to play an important role and obvious distinctions are seen for the three cases. We first compare the genuine SRT with the one without the $L$ valleys. As shown in Fig. 3(b), by removing the $L$ valleys in the calculation, surprisingly the SRT (curve with $\bullet$ ) becomes smaller instead of larger than its gen-
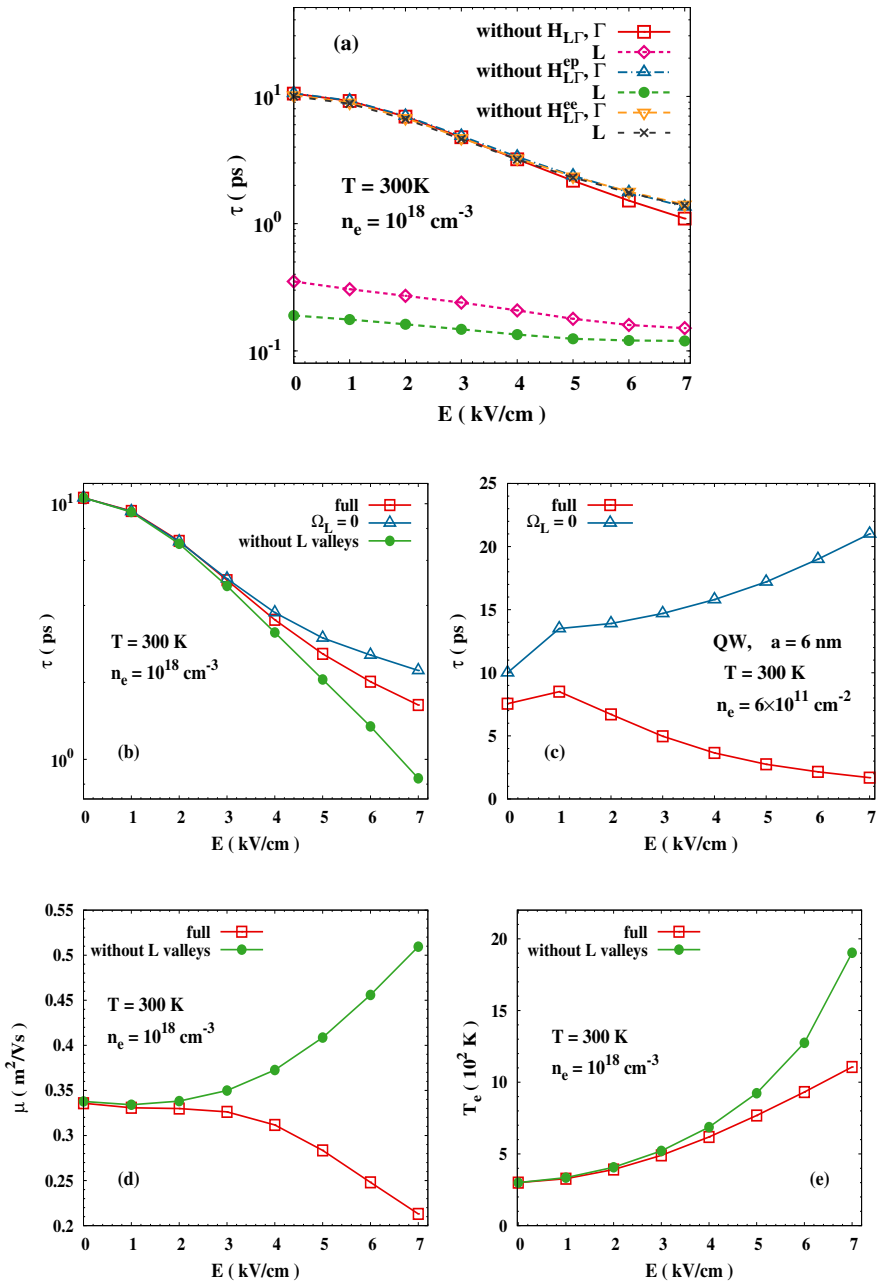

FIG. 3: (Color online) For (a), (b), (d) and (e): in bulk with electron density $n_{e}=10^{18} \mathrm{~cm}^{-3}$ : (a) SRTs $\tau$ of the $\Gamma$ and $L$ valleys as function of electric field $E$ by only removing the intervalley scattering $H_{L \Gamma}$ (curves with $\square$ and $\diamond$ ), the intervalley electron-phonon scattering $H_{L \Gamma}^{\mathrm{ep}}$ (curves with $\triangle$ and $\bullet$ ) or the intervalley electron-electron Coulomb scattering $H_{L \Gamma}^{\mathrm{ee}}$ (curves with $\nabla$ and $\times$ ). (b) SRT $\tau$ vs. electric field $E$ in the genuine condition [Case (i), curve with $\square$ ], by setting the SOC in the $L$ valleys to zero [Case (iii), curve with $\triangle$ ] and without the $L$ valleys [Case (ii), curve with •]. (d) and (e) Mobility $\mu$ and hot-electron temperature $T_{\Gamma} v s$. electric field with [Case (i), curve with $\square$ ] and without [Case (ii), curve with •] the $L$ valleys. For (c) in quantum well (QW) with well width $a=6 \mathrm{~nm}$ and $n_{e}=6 \times 10^{11} \mathrm{~cm}^{-2}$, SRT $\tau$ vs. electric field $E$ in the genuine condition [Case (i), curve with $\square$ ], and by setting the SOC in the $L$ valleys to zero [Case (iii), curve with $\triangle$ ]. $T=300 \mathrm{~K}$ in all the situations.

uine value (curve with $\square$ ). For $E=7 \mathrm{kV} / \mathrm{cm}$, the SRT without the $L$ valleys reaches down to half of the genuine value. In order to understand this behavior, we calculate the mobility and the hot-electron temperature $T_{\Gamma}$, which serve as scales of the drift and hot-electron effect, respectively. From Fig. 3(d) and (e), pronounced distinctions 
are seen for $E>4 \mathrm{kV} / \mathrm{cm}$. In the case without the $L$ valleys, the mobility increases rather than decreases with the electric field and reaches more than twice of its genuine value at $E=7 \mathrm{kV} / \mathrm{cm}$. Meanwhile, the hot-electron temperature $T_{\Gamma}$ reaches almost twice of its genuine value. Consequently, both the drift and hot-electron effects are markedly enhanced without the $L$ valleys, which in turn leads to a drastic increase of the inhomogeneous broadening due to the cubic form the $\Gamma$-valley Dresselhaus SOC in bulk. It overtakes the effect of the absence of the $L$ valleys and leads to the smaller SRT compared to the case with the $L$ valleys. This tells us that the $L$ valleys serve as a "momentum damping area" where electrons from the $\Gamma$ valley are blocked from reaching higher momentum states, thanks to the large effective mass in the $L$ valleys.

We then focus on the curves corresponding to Case (i) (curve with $\square$ ) and (iii) (curve with $\triangle$ ). The SRT calculated by setting the SOC in the $L$ valleys to zero becomes a little larger and reaches less than $3 / 2$ of the corresponding genuine value at $E=7 \mathrm{kV} / \mathrm{cm}$, indicating that the spin relaxation in the $L$ valleys only slightly modulates the total spin relaxation. This is very different from the quantum well system. As a comparison, in Fig. 3(c) we plot the SRTs against electric field in a quantum well in Case (i) and (iii) ${ }^{35,46}$ The well width is chosen as $6 \mathrm{~nm}$ and the two-dimensional electron density is $n_{e}=6 \times 10^{11} \mathrm{~cm}^{-2}$. It is seen that the behavior of field dependence of the SRT is totally changed in the absence of the SOC in the $L$ valleys. Instead of decreasing rapidly after a small increase, the SRT with $\boldsymbol{\Omega}_{L}=0$ increases monotonically and reaches more than ten times of the corresponding genuine value at $E=7 \mathrm{kV} / \mathrm{cm}$.

The underlying physics is understood as follows: in bulk, due to the cubic form of the Dresselhauss SOC in the $\Gamma$ valley, the field-induced drift effect and the hotelectron effect lead to a stronger enhancement of the inhomogeneous broadening compared to the increase of momentum scattering. Moreover, a simple calculation shows that although in average, the inhomogeneous broadening in the $\Gamma$ valley is much smaller than that in the $L$ valleys, in the energy range of the $\Gamma$ valley overlapping with the $L$ valleys (which is roughly where the exchange of electrons between the $\Gamma$ and $L$ valleys happens), the effective magnetic field from the Dresselhauss SOC is already comparable with that in the $L$ valleys. However, in quantum wells with a small well width, the enhancement of scattering is more effective than that of the inhomogeneous broadening thanks to the linear form of the Dresselhaus SOC ${ }^{35}$ Besides, the effective magnetic field in the energy range of the $\Gamma$ valley overlapping with the $L$ valleys is still much smaller than that in the $L$ valleys. In addition, it is further noted that electrons are more liable to be drifted into the $L$ valleys in quantum wells. In Fig. 33(c), $n_{L} / n_{e}$ reaches $45 \%$ at $E=7 \mathrm{kV} / \mathrm{cm}$ compared to $25 \%$ in bulk, which further enhances the effect of the $L$ valleys in quantum wells. Nevertheless, in Fig. 3)(c), the electric field corresponding to $n_{L} / n_{e}=0.25$ is $E=5 \mathrm{kV} / \mathrm{cm}$, at which the SRT without the SOC in the $L$ valleys is still about five times larger than the corresponding genuine value. All these lead to the pronounced difference between two- and three-dimensional systems. It also tells us that the $L$ valleys no longer serve as "spin drain" in bulk, differing from the case of quantum wells. 35

\section{B. Density dependence}

Another interesting feature seen in Fig. 2 is that the $\tau$ - $E$ curve corresponding to the electron density $n_{e}=$ $10^{18} \mathrm{~cm}^{-3}$ intersects with the other two, indicating that the density dependence of the SRT changes with the variation of electric field. In order to elucidate this behavior, we calculate the SRT by varying the electron density $n_{e}$ from $2 \times 10^{15}$ to $10^{18} \mathrm{~cm}^{-3}$ with electric field $E=0,4$ and $6 \mathrm{kV} / \mathrm{cm}$. Note that in bulk the impurity density $n_{i}$ is always taken as $n_{e}$ in this paper. From Fig. 4, one observes that for $E=0 \mathrm{kV} / \mathrm{cm}$, there is a peak at around $n_{e}=2.0 \times 10^{17} \mathrm{~cm}^{-3}$. This peak in the density dependence of the SRT has been theoretically predicted ${ }^{20}$ and experimentally confirmed ${ }^{13-15,54}$ very recently, and is attributed to the crossover from the nondegenerate to degenerate limit when the electron-impurity and electronelectron Coulomb scatterings are the dominant scattering processes 20 The crossover between degenerate and nondegenerate limits can be estimated by the Fermi temperature $T_{F}\left[=\left(3 \pi^{2} n_{e}\right)^{2 / 3} /(2 m)\right]$, with the peak determined by $T_{F} \sim \frac{1}{2} T-T, 13-15,20,47-49$ Here the Fermi temperature corresponding to the electron density at the peak is $T_{F} \approx 207 \mathrm{~K}$, comparable to the lattice temperature $T=300 \mathrm{~K}\left(T_{F} / T \sim 2 / 3\right)$, in line with the previous works.

By applying an electric field $E=4 \mathrm{kV} / \mathrm{cm}$, one observes from Fig. 4 that the peak moves to around $n_{e}=5 \times 10^{17} \mathrm{~cm}^{-3}$, and when the electric field is further increased to $E=6 \mathrm{kV} / \mathrm{cm}$, the peak is shifted to even higher electron density (at around $n_{e}=9 \times 10^{17} \mathrm{~cm}^{-3}$ hence is not very obvious in the figure). We point out that this is due to the increase of hot-electron temperature induced by the strengthened electric field. As

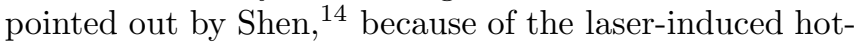
electron effect, the peak of density dependence of the SRT appears at where the hot-electron temperature $T_{e}$, rather than the lattice temperature $T$, approximately equals the Fermi temperature $T_{F}$. The underlying physics is similar here with the electric-field-induced hot-electron effect. However, as shown in Fig. [6 in Appendix B, the $L$ valleys start to play a role with high electric field and moreover, the two-hot-electron-temperature behavior of the $\Gamma$-valley electrons is seen. This makes the situation more complex. Nevertheless, by comparing the curves of "intrinsic" SRTs of the $\Gamma$ and $L$ valleys in Fig. 3(a) with the corresponding genuine one in Fig. 3(b), one notices that the curve of the "intrinsic" SRT in the $\Gamma$ valley resembles the genuine one while that of the "intrinsic" SRT in the $L$ valleys is far away it. This indicates that the 
the multivalley spin relaxation in the presence of high electric field is mainly determined by the $\Gamma$ valley. Furthermore, since most electrons stay in the $\Gamma$ valley for electric field up to $6 \mathrm{kV} / \mathrm{cm}$ [see Fig. 6(c) in Appendix B] and these electrons mostly distribute in the lower-energy regime compared to the high-energy regime overlapping the $L$ valleys, the behavior of the SRT is mainly determined by this part of electrons. In the approximation that all electrons are in the $\Gamma$ valley, for $E=4 \mathrm{kV} / \mathrm{cm}$, we have $T_{F} \approx 381 \mathrm{~K}$ according to the electron density at the peak, comparable to the corresponding hot-electron temperature $T_{e} \approx 659 \mathrm{~K}\left(T_{F} / T_{e} \sim 3 / 5\right)$. Meanwhile for $E=6 \mathrm{kV} / \mathrm{cm}$, we have $T_{F} \approx 563 \mathrm{~K}$ compared to $T_{e} \approx 981 \mathrm{~K}$ at the peak $\left(T_{F} / T_{e} \sim 3 / 5\right)$. We note that this effect of electric field on the density dependence of the SRT can be observed within current technology of optical orientation.

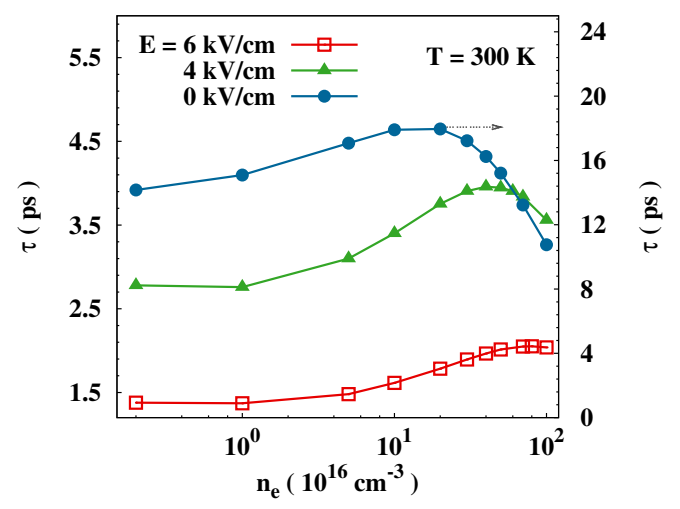

FIG. 4: (Color online) SRT $\tau$ vs. electron density at temperature $T=300 \mathrm{~K}$ for fixed electric field: $E=0,4$ and $6 \mathrm{kV} / \mathrm{cm}$. Note that the scale of the case $E=0 \mathrm{kV} / \mathrm{cm}$ is on the right hand side of the frame.

\section{Temperature dependence}

We now turn to investigate the temperature dependence of the SRT. In Fig. 5, the SRTs obtained are plotted as function of lattice temperature with electric field $E=0,4$ and $6 \mathrm{kV} / \mathrm{cm}$ and for three electron densities as in Sec. IIIA. In the field-free case, it has been formerly shown both experimentally ${ }^{10,18,19,50}$ and theoretically ${ }^{20.51}$ that in $n$-type samples with low mobility, the SRT decreases monotonically with temperature. From Fig. [5(a), it is seen that our result coincides with the previous ones. However, very different behaviors are seen under high electric fields. Apart from the overall suppressed values of the SRT compared to the field-free condition, it is found that for electron density $n_{e}=10^{18} \mathrm{~cm}^{-3}$, the decreasing rate becomes smaller for higher electric fields. Whereas for $n_{e}=10^{17} \mathrm{~cm}^{-3}$, the
SRT turns to increase with increasing temperature under electric field $E=6 \mathrm{kV} / \mathrm{cm}$. The most interesting phenomenon is seen for the case with $n_{e}=10^{16} \mathrm{~cm}^{-3}$. From Fig. [5(a)-(c), one observes that the SRT decreases monotonically with increasing lattice temperature $T$ in the absence of the electric field but increases monotonically with it when electric field $E=6 \mathrm{kV} / \mathrm{cm}$ is applied. In between, for the case with $E=4 \mathrm{kV} / \mathrm{cm}$, the SRT first increases and then decreases with increasing temperature $T$, with a peak at around $T=350 \mathrm{~K}$. This peak in the temperature dependence of the SRT is very different from the one theoretically predicted by Zhou et $a l^{\underline{51}}$ and experimentally realized by Leyland et al., 52 Ruan et al 53 and Han et al $\stackrel{54}{\underline{4}}$ for high-mobility samples in the fieldfree condition. There, the peak is solely caused by the electron-electron Coulomb scattering $38,51-58$ and appears in the crossover between the degenerate and nondegenerate limits where the Fermi temperature $T_{F}$ is comparable to the lattice temperature $\sqrt[51-54]{ }$ However, in $n$-type bulk materials, due to the strong electron-impurity scattering (hence low mobility), the Coulomb scattering is always less important and no peak is expected in the temperature dependence of the SRT $\underline{\underline{18}-20}$ Moreover, the Fermi temperature corresponding to $n_{e}=10^{16} \mathrm{~cm}^{-3}$ is $T_{F} \approx 28 \mathrm{~K}$, which is far below the lattice temperature, let alone the hot-electron temperature under the high electric field. This further demonstrates the essential difference of the peak observed here.

This complex behavior of the SRT can be understood from the different lattice-temperature dependences of the hot-electron temperature at the different electron densities and electric fields. Corresponding to Fig. 5 (b) and (c), in Fig. 5(d) and (e) we plot the hot-electron temperatures against the lattice temperature for $E=4$ and $6 \mathrm{kV} / \mathrm{cm}$, respectively. Note that the same color and type of point are used for the corresponding electron density. By comparing the $T_{\Gamma}-T$ curves in Fig. [5(b) with the $\tau-T$ ones in Fig. 5(d) and those in Fig. 5(c) with Fig. 5 (e), direct correspondence of the temperature dependence of the SRT to that of the hot-electron temperature $T_{\Gamma}$ is observed. The order of curves from top to bottom reverses in the corresponding two figures as higher hot-electron temperature indicates larger inhomogeneous broadening, and hence smaller SRT. Meanwhile, the decrease (increase) of the hot-electron temperature gives an increase (decrease) of the SRT in the lattice temperature dependence. Specifically, the monotonic decrease (increase) of the SRT with $n_{e}=10^{18} \mathrm{~cm}^{-3}\left(n_{e}=10^{16} \mathrm{~cm}^{-3}\right)$ in Fig. 5(b) [Fig. 5(c)] corresponds to the monotonic increase (decrease) of the hot-electron temperature in Fig. 5(d) [Fig. 5(e)]. Especially corresponding to the peak in Fig. 5(b) for $n_{e}=10^{16} \mathrm{~cm}^{-3}$, there is a valley in the lattice-temperature dependence of the hot-electron temperature in Fig. 5(d). For the electric field-free case, the electron temperature equals the lattice temperature, so the behavior of the SRT in Fig. 5(a) is also in the same trend as the high field cases. It is noted that in the discussion above, we focus on $T_{\Gamma}$ instead of $T_{L}$. This 

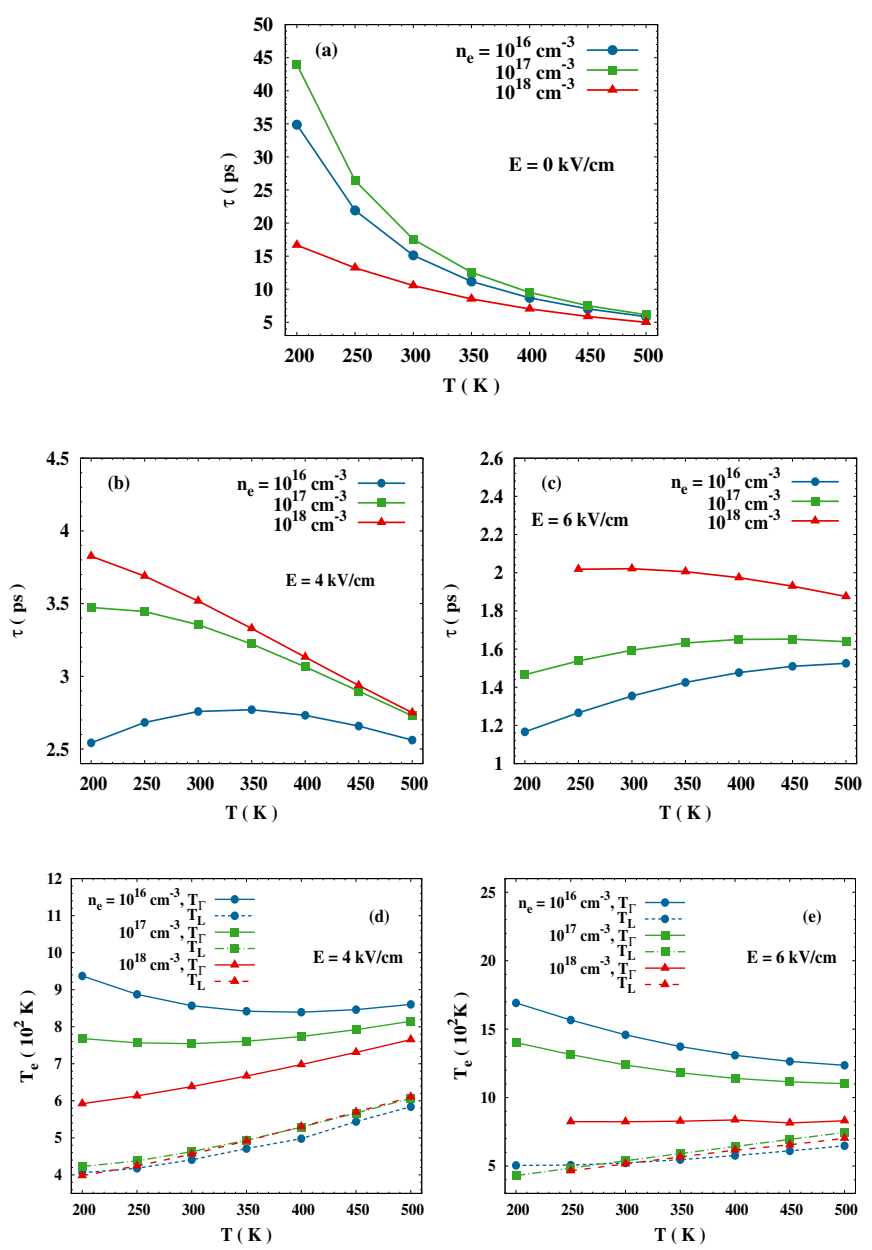

FIG. 5: (Color online) SRT $\tau$ vs. lattice temperature $T$ with (a) $E=0 \mathrm{kV} / \mathrm{cm}$; (b) $E=4 \mathrm{kV} / \mathrm{cm}$ and (c) $E=6 \mathrm{kV} / \mathrm{cm}$. Hot-electron temperatures $T_{\Gamma}$ and $T_{L}$ vs. lattice temperature $T$ with (d) $E=4 \mathrm{kV} / \mathrm{cm}$ and (e) $E=6 \mathrm{kV} / \mathrm{cm}$. The electron densities are $n_{e}=10^{16}, 10^{17}$ and $10^{18} \mathrm{~cm}^{-3}$ in these figures.

is because, as discussed in the previous subsection, the total spin relaxation is mainly determined by the $\Gamma$ valley while most $\Gamma$-valley electrons stay in the lower-energy regime. Meanwhile, according to the the discussions in Sec. IIIA, in the variation of the hot-electron temperature, the variation of inhomogeneous broadening is more profound than that of the momentum relaxation, thanks to the cubic $\mathbf{k}$ dependence of the $\Gamma$-valley Dresselhauss SOC 43,44 Therefore the variation of the inhomogeneous broadening mainly determines the variation of the SRT.

We point out that the different behaviors of hotelectron temperature with the lattice temperature at different electron densities and electric fields are originated from the temperature dependence of the energygain and loss rates of the electron system. ${ }^{64}-67$ With the increase of lattice temperature, the mobility is reduced resulting from the enhancement of scattering, so does the the energy-gain rate; meanwhile the energy-loss rate decreases due to increase of phonon number ${ }^{64-67}$ These two effects compete with each other and lead to the complex behavior of the hot-electron temperature in different conditions. A more detailed discussion is given in Appendix C.

\section{SUMMARY}

In summary, we have investigated the multivalley spin relaxation in $n$-type bulk GaAs in the presence of high electric field by applying the fully microscopic KSBE approach. The $\Gamma$ and $L$ valleys, which are relevant in determining the properties of spin dynamics for the high electric field applied in this work, are taken into account. The effect of the $L$ valleys on spin relaxation is discussed and is shown to be very different from the quantum well system. We find that apart from the effect of directly manipulating the SRT, the high electric field can also effectively modulate the density and temperature dependences of the SRT.

First, the SRT is found to decrease monotonically with the electric field for electron densities from the nondegenerate to degenerate limit. This monotonic field dependence of the SRT is very different from the previous works in $n$-type GaAs quantum wells ${ }^{33-35}$ and is assigned to the pronounced enhancement of inhomogeneous broadening from the field-induced drift and hot-electron effects thanks to the cubic form of the $\Gamma$-valley Dresselhauss SOC in bulk. We show that, in despite of the very different strength of the SOC in different valleys, the evolutions of spin polarizations in the $\Gamma$ and $L$ valleys share the same damping rate. This is demonstrated to come from the strong intervalley electron-phonon scattering and indicates the feasibility of exploring the properties of the hot electrons in the $L$ valleys through the phenomena detected in the $\Gamma$ valley. Moreover, differing from the role of a "spin drain" of the total spin polarization in the two-dimensional system, we find that in bulk the $L$ valleys serve as a "momentum damping area" where electrons are blocked from drifting to higher momentum states due to the large effective mass. This tends to suppress the inhomogeneous broadening and in turn leads to a longer rather than shorter SRT compared to the case without the $L$ valleys.

As for the density dependence of the SRT, the formerly predicted ${ }^{20}$ and experimentally observed ${ }^{13,15}$ density peak in the field-free condition is recovered and is found to be shifted to higher density regime with higher electric field. We attribute this to the electric-fieldinduced hot-electron effect.

We also investigate the temperature dependence of the SRT in conditions with different electron densities and electric fields. The monotonic decrease of the SRT with increasing lattice temperature in the field-free condition coincides with the previous works. Nevertheless, the SRT is found to decrease more slowly with higher electric field, and even turn to increase monotonically with increasing lattice temperature in the condition with low electron 
density and high electric field. More interestingly, a peak is predicted in the temperature dependence with low electron density and moderate electric field, which is vastly different from the formerly discussed one in high mobility samples in the field-free condition. We point out that this peculiar behavior of the SRT originates from the temperature dependence of the energy-gain and loss rates of the electron system.

Finally, we remark on the feasibility of the spin Gunn effect in $n$-type bulk GaAs. For the preferred electron density in Ref. $37\left(n_{e}=10^{18} \mathrm{~cm}^{-3}\right)$, we note that the SRT is suppressed down to the value shorter than what required for the spontaneous spin amplification to appear under the electric fields where the charge Gunn effect appears. This fast damping of spin polarization overtakes the spontaneously generation process and makes the spin Gunn effect hardly to be realized in $n$-type bulk GaAs system.

\section{Acknowledgments}

This work was supported by the National Natural Science Foundation of China under Grant No. 10725417 and the National Basic Research Program of China under Grant No. 2012CB922002. Discussions with X. Marie is acknowledged. We also would like to thank P. Zhang for calculating the SRTs in quantum wells in Fig. B(c). One of the authors (H.T.) would like to thank K. Shen for valuable discussions.

\section{Appendix A: Scattering Terms in KSBEs}

The scattering term $\left.\partial_{t} \rho_{\lambda \mathbf{k}_{\lambda}}\right|_{\text {scat }}$ includes the contributions from the electron-impurity scattering $\left.\partial_{t} \rho_{\lambda \mathbf{k}_{\lambda}}\right|_{\mathrm{ei}}$, the electron-phonon scattering $\left.\partial_{t} \rho_{\lambda \mathbf{k}_{\lambda}}\right|_{\text {ep }}$ and the electronelectron Coulomb scattering $\left.\partial_{t} \rho_{\lambda \mathbf{k}_{\lambda}}\right|_{\mathrm{ee}}$

$$
\left.\partial_{t} \rho_{\lambda \mathbf{k}_{\lambda}}\right|_{\text {scat }}=\left.\partial_{t} \rho_{\lambda \mathbf{k}_{\lambda}}\right|_{\mathrm{ei}}+\left.\partial_{t} \rho_{\lambda \mathbf{k}_{\lambda}}\right|_{\mathrm{ep}}+\left.\partial_{t} \rho_{\lambda \mathbf{k}_{\lambda}}\right|_{\mathrm{ee}}
$$

where

$$
\begin{aligned}
& \left.\partial_{t} \rho_{\lambda \mathbf{k}_{\lambda}}\right|_{\mathrm{ei}} \\
& =-\pi n_{i} Z_{i}^{2} \sum_{\mathbf{k}_{\lambda}^{\prime}} V_{\mathbf{k}_{\lambda}-\mathbf{k}_{\lambda}^{\prime}}^{2} \delta\left(\varepsilon_{\mathbf{k}_{\lambda}^{\prime}}^{\lambda}-\varepsilon_{\mathbf{k}_{\lambda}}^{\lambda}\right) \\
& \times\left(\rho_{\lambda \mathbf{k}_{\lambda}^{\prime}}^{>} \rho_{\lambda \mathbf{k}_{\lambda}}^{<}-\rho_{\lambda \mathbf{k}_{\lambda}^{\prime}}^{<} \rho_{\lambda \mathbf{k}_{\lambda}}^{>}\right)+\text {h.c. } \\
& \left.\partial_{t} \rho_{\lambda \mathbf{k}_{\lambda}}\right|_{\mathrm{ep}} \\
& =-\pi \sum_{\lambda^{\prime}, \mathbf{k}_{\lambda^{\prime}}^{\prime}, \pm}\left|M_{\lambda \lambda^{\prime}, \mathbf{k}_{\lambda}-\mathbf{k}_{\lambda^{\prime}}^{\prime}}\right|^{2} \delta\left( \pm \Omega_{\lambda \lambda^{\prime}}+\varepsilon_{\mathbf{k}_{\lambda^{\prime}}^{\prime}}^{\lambda^{\prime}}-\varepsilon_{\mathbf{k}_{\lambda}}^{\lambda}\right) \\
& \times\left(N_{\lambda \lambda^{\prime}}^{ \pm} \rho_{\lambda^{\prime} \mathbf{k}_{\lambda^{\prime}}^{\prime}}^{>} \rho_{\lambda \mathbf{k}_{\lambda}}^{<}-N_{\lambda \lambda^{\prime}}^{\mp} \rho_{\lambda^{\prime} \mathbf{k}_{\lambda^{\prime}}^{\prime}}^{<} \rho_{\lambda \mathbf{k}_{\lambda}}^{>}\right)+\text {h.c., (A3) } \\
& \left.\partial_{t} \rho_{\lambda \mathbf{k}_{\lambda}}\right|_{\mathrm{ee}} \\
& =-\pi \sum_{\lambda^{\prime}, \mathbf{k}_{\lambda}^{\prime}, \mathbf{k}_{\lambda^{\prime}}^{\prime \prime}} \delta\left(\varepsilon_{\mathbf{k}_{\lambda}^{\prime}}^{\lambda}-\varepsilon_{\mathbf{k}_{\lambda}}^{\lambda}+\varepsilon_{\mathbf{k}_{\lambda^{\prime}}^{\prime \prime}}^{\lambda^{\prime}}-\varepsilon_{\mathbf{k}_{\lambda^{\prime}}^{\prime \prime}-\mathbf{k}_{\lambda}+\mathbf{k}_{\lambda}^{\prime}}^{\lambda^{\prime}}\right) \\
& \times V_{\mathbf{k}_{\lambda}-\mathbf{k}_{\lambda}^{\prime}}^{2}\left[\rho_{\lambda \mathbf{k}_{\lambda}^{\prime}}^{>} \rho_{\lambda \mathbf{k}_{\lambda}}^{<} \operatorname{Tr}\left(\rho_{\lambda^{\prime}\left(\mathbf{k}_{\lambda^{\prime}}^{\prime \prime}-\mathbf{k}_{\lambda}+\mathbf{k}_{\lambda}^{\prime}\right)}^{<} \rho_{\lambda^{\prime} \mathbf{k}_{\lambda^{\prime}}^{\prime \prime}}^{>}\right)\right. \\
& \left.-\rho_{\lambda \mathbf{k}_{\lambda}^{\prime}}^{<} \rho_{\lambda \mathbf{k}_{\lambda}}^{>} \operatorname{Tr}\left(\rho_{\lambda^{\prime}\left(\mathbf{k}_{\lambda^{\prime}}^{\prime \prime}-\mathbf{k}_{\lambda}+\mathbf{k}_{\lambda}^{\prime}\right)}^{>} \rho_{\lambda^{\prime} \mathbf{k}_{\lambda^{\prime}}^{\prime \prime}}^{<}\right)\right]+ \text {h.c.(A }
\end{aligned}
$$

In these equations, $\rho_{\mathbf{k}}^{<}=\rho_{\mathbf{k}}$ and $\rho_{\mathbf{k}}^{>}=1-\rho_{\mathbf{k}}$. $n_{i}$ is the impurity density which equals the electron density in this paper and $Z_{i}=1$ is the charge number of the impurity. $\varepsilon_{\mathbf{k}_{\Gamma}}^{\Gamma}=k_{\Gamma}^{2} /\left(2 m_{\Gamma}^{*}\right)$ and $\varepsilon_{\mathbf{k}_{L_{i}}}^{L_{i}}=k_{L_{i}}^{2} /\left(2 m_{L}^{*}\right)+\varepsilon_{\Gamma L}$ with $\varepsilon_{\Gamma L}$ denoting the energy difference between the $\Gamma$ and $L$ points. $N_{\lambda \lambda^{\prime}}=\left[e^{\left(\Omega_{\lambda \lambda^{\prime}} / T\right)}-1\right]^{-1}$ is the Bose distribution of phonons with frequency $\Omega_{\lambda \lambda^{\prime}} . N_{\lambda \lambda^{\prime}}^{<}=N_{\lambda \lambda^{\prime}}$ and $N_{\lambda \lambda^{\prime}}^{>}=1+N_{\lambda \lambda^{\prime}} . \quad M_{\lambda \lambda^{\prime}, \mathbf{q}}$ is the matrix element of the electron-phonon scattering with $\mathbf{q}$ standing for the phonon wave vector. Here we take into account the intra- and intervalley electron-longitudinal-optical (LO) phonon scattering in and between the $\Gamma$ and $L$ valleys, respectively $\underline{35,41,59} \underline{\underline{62}}$ For the intravalley electronphonon scattering, we have $M_{\Gamma \Gamma, \mathbf{q}}^{2}=\frac{e^{2} \Omega_{\Gamma \Gamma}\left(\kappa_{\infty}^{-1}-\kappa_{0}^{-1}\right)}{2 \epsilon_{0} q^{2}}$ and $M_{L_{i} L_{i}, \mathbf{q}}^{2}=\frac{D_{L_{i} L_{i}}^{2}}{2 d \Omega_{L_{i} L_{i}}}$. Also we have $M_{\Gamma L_{i}, \mathbf{q}}^{2}=M_{L_{i} \Gamma, \mathbf{q}}^{2}=$ $\frac{D_{\Gamma L}^{2}}{2 d \Omega_{\Gamma L}}$ for the $\Gamma-L$ intervalley electron-phonon scattering and $M_{L_{i} L_{j}, \mathbf{q}}^{2}=\frac{D_{L_{i} L_{j}}^{2}}{2 d \Omega_{L_{i} L_{j}}}$ for $L-L$ intervalley electronphonon scattering. $V_{q}$ is the screened Coulomb potential under the random phase approximation,, 63

$$
V_{\mathbf{q}}=\frac{V_{\mathbf{q}}^{(0)}}{1-V_{\mathbf{q}}^{(0)} P^{(1)}(\mathbf{q})},
$$

where

$$
P^{(1)}(\mathbf{q})=\sum_{\lambda, \mathbf{k}_{\lambda}, \sigma} \frac{f_{\lambda\left(\mathbf{k}_{\lambda}+\mathbf{q}\right), \sigma}-f_{\lambda \mathbf{k}_{\lambda}, \sigma}}{\varepsilon_{\mathbf{k}_{\lambda}+\mathbf{q}}^{\lambda}-\varepsilon_{\mathbf{k}_{\lambda}}^{\lambda}},
$$

with $V_{\mathbf{q}}^{(0)}=e^{2} /\left(\epsilon_{0} \kappa_{0} q^{2}\right)$ denoting the bare Coulomb potential and $f_{\lambda \mathbf{k}, \sigma}$ being the electron distribution of spin- $\sigma$ band. It is noted that in Eq. (A4), we include both the intra- and intervalley electron-electron Coulomb scattering. All parameters appear in these equations are listed in Table. I. 

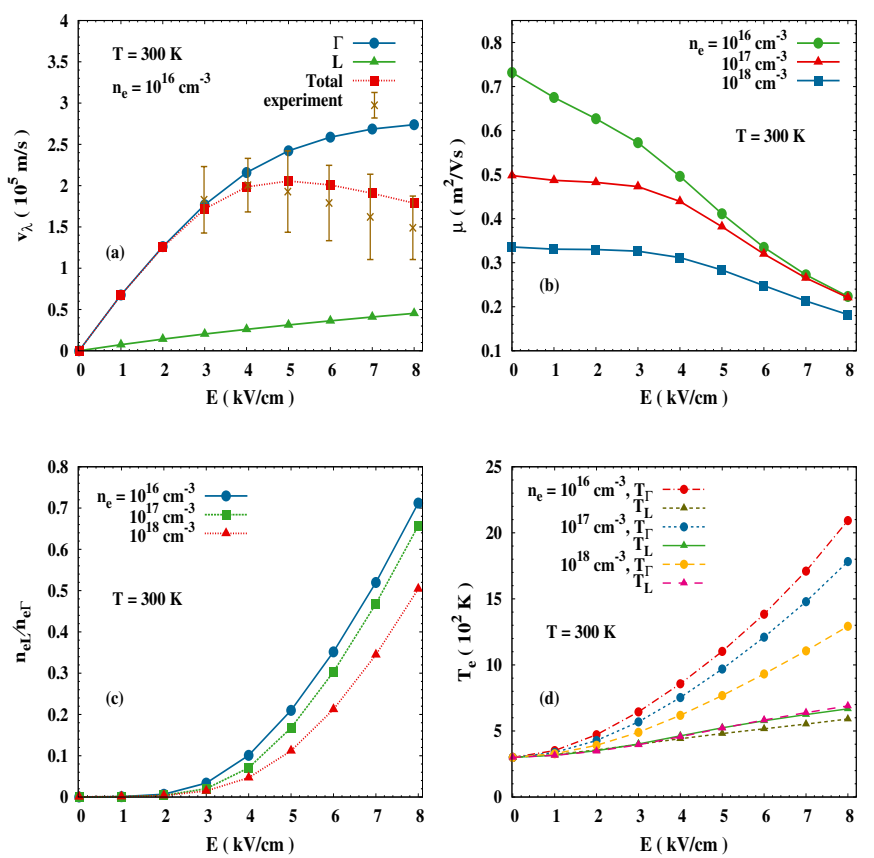

FIG. 6: (Color online) Electric field dependences of (a) drift velocity $v_{\lambda}$ for electron density $n_{e}=10^{16} \mathrm{~cm}^{-3}$, (b) mobility $\mu$, (c) ratio of electron densities in the $L$ and $\Gamma$ valleys and (d) hot-electron temperature for three electron densities $n_{e}=$ $10^{16}, 10^{17}$ and $10^{18} \mathrm{~cm}^{-3}$. The lattice temperature is $T=$ $300 \mathrm{~K}$ and the experimental data are taken from Ref. 32 .

\section{Appendix B: Drift velocity, Mobility, $L$-valley Occupation and Hot-electron Temperature under Electric Field}

In order to have an overview of the electric properties influenced by the high electric field, the steady-state drift velocity $v_{\lambda}$ of each valley as well as the total drift velocity are calculated by varying the electric field from 0 to $8 \mathrm{kV} / \mathrm{cm}$. In Fig. 6(a), we plot the drift velocity $v_{\lambda}$ as function of electric field with $n_{e}=10^{16} \mathrm{~cm}^{-3}$ and $T=300 \mathrm{~K}$. The negative differential electric conductance can be seen from the field dependence of the total drift velocity and good agreement is reached with the experimental results. $\underline{32}$ We also calculate the field dependences of the mobility and the high-valley electron population for three different doping densities. From Fig. 6(b), one finds that the mobilities first decrease slowly with the increase of electric field and then more rapidly when the electric field is increased over $E=4 \mathrm{kV} / \mathrm{cm}$. This can be understood with the help of Fig. 6(c) where the ratio of electron densities in the $\Gamma$ and $L$ valleys is plotted against the electric field $E$. It is seen that the population of electrons in the $L$ valleys is negligible when $E<2 \mathrm{kV} / \mathrm{cm}$ and approaches $10 \%$ when $E \sim 4 \mathrm{kV} / \mathrm{cm}$. This contributes to the faster decrease of mobilities in Fig. 6(b) and leads to the negative differential electric conductance in Fig. 6(a).
The hot-electron temperature $T_{e}$ is obtained by fitting the calculated steady-state electron distribution of each valley with the drifted Fermi distribution $f\left(\varepsilon_{\mathbf{k}_{\lambda}, E}\right)=$ $\left[\exp \left(\left(\varepsilon_{\mathbf{k}_{\lambda}, E}-\mu_{\lambda}\right) / T_{\lambda}\right)+1\right]^{-1}$. Here $\varepsilon_{\mathbf{k}_{\lambda}, E}=\left(\mathbf{k}_{\lambda}-\right.$ $\left.\mathbf{k}_{\lambda}^{0}\right)^{2} / 2 m_{\lambda}^{*}$ is the energy spectrum shifted by the electric field with $\mathbf{k}_{\lambda}^{0}$ being the drift momentum and $\mu_{\lambda}$ is a fitting parameter denoting the chemical potential in $\lambda$ valley. It is found that electrons in the $\Gamma$ valley carry two temperatures, one in the higher-energy regime that overlaps with the $L$ valleys (labeled as $T_{L}$ ) and the other in the lower-energy regime (labeled as $T_{\Gamma}$ ). Electrons in the $L$ valleys share the same temperature as those in the higher-energy regime of the $\Gamma$ valley due to the rapid exchange of electrons thanks to the strong intervalley scattering. In Fig. 6(d), we plot $T_{\Gamma}$ and $T_{L}$ against the electric field at lattice temperature $T=300 \mathrm{~K}$. It is seen that electrons in the $\Gamma$ valley are easier to be heated due to the smaller effective mass. Moreover, by increasing the electron density from $10^{16}$ to $10^{18} \mathrm{~cm}^{-3}, T_{\Gamma}$ is effectively reduced while $T_{L}$ stays almost unchanged. The underlying physics is that by increasing the electron density, the electron-impurity scattering is enhanced. This tends to reduce the drift velocity in each valley and suppress the ability of electrons to gain energy from the electric field, and therefore reduce the hot-electron temperature $64-67$ Since the electron-impurity scattering is the leading scattering in the $\Gamma$ valley $\stackrel{20}{\rightleftharpoons}$ whereas the intervalley electronphonon scattering is dominant in the $L$ valleys, $\frac{68}{}$ the drift velocity, and hence also the hot-electron temperature of the $L$ valleys, are less affected by the electron density compared to those of the $\Gamma$ valley.

\section{Appendix C: Energy-gain and Loss Rates}

For a semiconductor system under uniform electric field, the electrons accelerate before they are scattered and thus gain energy from the electric field. Meanwhile due to the electron-phonon scattering, the electrons transfer energy to the phonon system. In steady state, the electron energy-gain rate equals the energyloss one $\underline{\underline{64}-\underline{67}}$

We calculate the energy-gain and loss rates in $n$-type bulk GaAs by including only the $\Gamma$ valley. The energygain rate $\eta$ (in unit volume here and hereafter) reads 66

$$
\eta=e n_{e} \mu E^{2}
$$

and the energy-loss rate $\omega$ is given by $\underline{67}$

$$
\begin{aligned}
\omega= & 2 \sum_{\mathbf{q}, \chi} \Omega_{\mathbf{q}, \chi}|M(\mathbf{q}, \chi)|^{2} \Pi_{2}\left(\mathbf{q}, \Omega_{\mathbf{q}, \chi}+\omega_{0}\right) \\
& \times\left[n\left(\frac{\Omega_{\mathbf{q}, \chi}}{T}\right)-n\left(\frac{\Omega_{\mathbf{q}, \chi}+\omega_{0}}{T_{e}}\right)\right],
\end{aligned}
$$

with

$$
\begin{aligned}
\Pi_{2}(\mathbf{q}, \omega)= & 2 \pi \sum_{\mathbf{k}}\left[f\left(\varepsilon_{\mathbf{k}}, T_{e}\right)-f\left(\varepsilon_{\mathbf{k}+\mathbf{q}}, T_{e}\right)\right] \\
& \times \delta\left(\varepsilon_{\mathbf{k}+\mathbf{q}}-\varepsilon_{\mathbf{k}}+\omega\right) .
\end{aligned}
$$


In these equations, $\Omega_{\mathbf{q}, \chi}$ is the phonon energy with momentum $\mathbf{q}$ and mode $\chi$. Note that here we only need to take account of the intravalley electron-LO phonon scattering with $\left|M_{\Gamma \Gamma, \mathbf{q}}\right|^{2}$ given the Appendix A. $\omega_{0}=$ $\mathbf{q} \cdot \mathbf{v}_{d}$ with $\mathbf{v}_{d}$ denoting the drift velocity. $n(x)=$
$1 /\left(e^{x}-1\right)$ stands for the Bose distribution and $f\left(x, T_{e}\right)=$ $1 /\left[e^{(x-\mu) / T_{e}}+1\right]$ is the Fermi distribution with $T_{e}$ and $\mu$ being the electron temperature and the chemical potential, respectively.
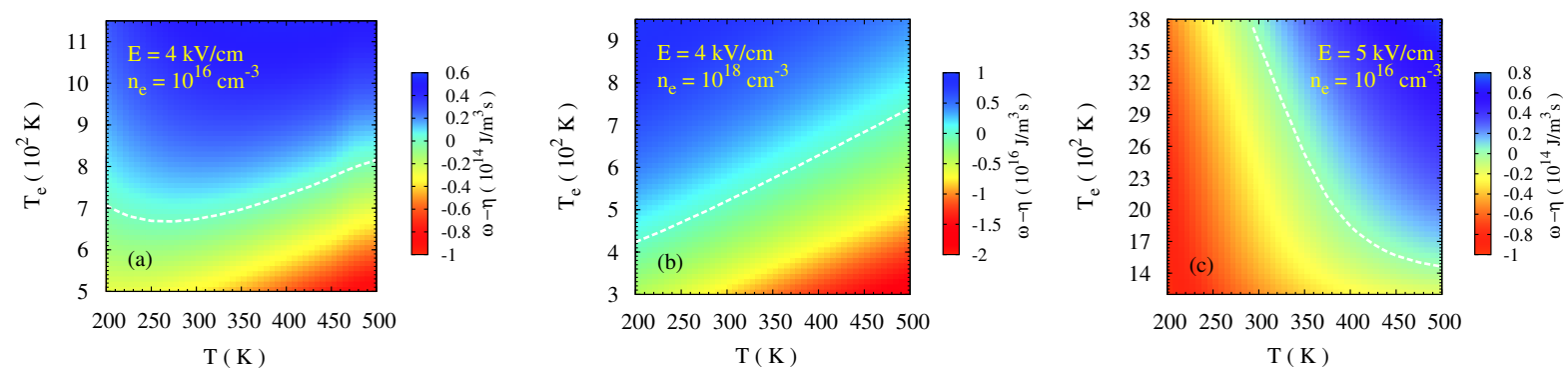

FIG. 7: (Color online) The difference of energy-gain and loss rates $\omega-\eta$ against the lattice temperature and hot-electron temperature. (a) $n_{e}=10^{16} \mathrm{~cm}^{-3}$ and $E=4 \mathrm{kV} / \mathrm{cm}$; (b) $n_{e}=10^{18} \mathrm{~cm}^{-3}$ and $E=4 \mathrm{kV} / \mathrm{cm} ;(\mathrm{c}) n_{e}=10^{16} \mathrm{~cm}{ }^{-3}$ and $E=5 \mathrm{kV} / \mathrm{cm}$. The dashed curves indicate the points where $\omega=\eta$, hence are just the plots of $T_{e}-T$ in equilibrium.

From Eqs. (C1) and (C2), we calculate the energy-gain and loss rates in three cases: (i) $n_{e}=10^{16} \mathrm{~cm}^{-3}$ with $E=4 \mathrm{kV} / \mathrm{cm}$; (ii) $n_{e}=10^{18} \mathrm{~cm}^{-3}$ with $E=4 \mathrm{kV} / \mathrm{cm}$ and (iii) $n_{e}=10^{16} \mathrm{~cm}^{-3}$ with $E=5 \mathrm{kV} / \mathrm{cm}$. The lattice temperature is varied in the range $T=200 \sim 500 \mathrm{~K}$ and the hot-electron temperature $T_{e}$ in the proper range according to the electron density and the electric field. The drift velocity used in the calculation are obtained by solving the KSBEs. In Fig. 7, we plot $\omega-\eta$ against $T$ and $T_{e}$ for the three cases. The points where $\omega-\eta=0$ are indicated with dashed curve, which is exactly the hotelectron temperature $T_{e}$ versus to the lattice temperature $T$ in the steady state. By comparing the dashed curves in Fig. 7(a) and (b) with the corresponding curves in Fig. 5(d), one notices that qualitatively, good agreement is reached. We note that for case (iii), due to the large electric field, this model does not hold so well in describing the genuine system since the $L$ valleys start to play an important role and serve as the "momentum damping area" where electrons are hardly drifted. Therefore in the simplified model, the drift effect is overestimated and the intervalley electron-phonon scatterings, which serve as additional energy-loss channels, are missing. These lead to the overestimation of $T_{e}$. However, the qualitative behavior of hot-electron temperature with the lattice temperature in the presence of extremely high electric field is still captured: $T_{e}$ decreases with increasing $T$.

The different behaviors of $T_{e}$ with $T$ can be understood as follows. The increase of the lattice tempera- ture induces two main effects: (1) It reduces the mobility by enhancing the electron-phonon scattering, which in turn leads to the decrease of the energy-gain rate [see Eq. [C1)]. This tends to reduce the hot-electron temperature. (2) It also modulates the relative importance of phonon-emitting and absorbing processes in the electronphonon scattering and reduces the energy-loss rate for the electron system (by reducing the temperature difference between the electron and phonon systems). This tends to "heat" the electron system. The competing of these two factors contributes to the complex $T$-dependence of $T_{e}$, hence also that of the SRT. In Fig. [7 we have shown three typical cases investigated. In Fig. 77(c) with high electric field and low electron density, the hot-electron temperature $T_{e}$ is high above the lattice temperature $T$. As a result, the small increase in $T$ does not affect the energy-loss rate much but does enhance the scattering. This results in the leading role of the reduction of the energy-gain rate, which in turn leads to the decrease of $T_{e}$. Whereas in Fig. 7(b) where $T_{e}$ is much closer to $T$ compared to that in Fig. 77(c), by increasing the lattice temperature, the temperature difference between the electron and phonon systems is effectively reduced and hence the "heating" effect is more efficient. This leads to the increase of the electron temperature. In between, for the case with relatively low electric field and low electron density, a nonmonotonic temperature dependence is expected, which is just the case in Fig. 7(a). 
* Author to whom correspondence should be addressed; Electronic address: mwwu@ustc.edu.cn.

${ }^{1}$ F. Meier and B. P. Zakharchenya, Optical Orientation (North-Holland, Amsterdam, 1984).

2 S. A. Wolf, D. D. Awschalom, R. A. Buhrman, J. M. Daughton, S. Von Molnár, M. L. Roukes, A. Y. Chtchelkanova, and D. M. Treger, Science 294, 1488 (2001).

3 Semiconductor Spintronics and Quantum Computation, ed. by D. D. Awschalom, D. Loss, and N. Samarth (Springer-Verlag, Berlin, 2002).

4 I. Žutić, J. Fabian, and S. Das Sarma, Rev. Mod. Phys. 76, 323 (2004).

5 J. Fabian, A. Matos-Abiague, C. Ertler, P. Stano, and I. Žutić, Acta Phys. Slov. 57, 565 (2007).

6 Spin Physics in Semiconductors, ed. by M. I. D'yakonov (Springer, Berlin, 2008).

7 M. W. Wu, J. H. Jiang, and M. Q. Weng, Phys. Rep. 493, 61 (2010).

8 Handbook of Spin Transport and Magnetism, ed. by E. Y. Tsymbal and I. Žutić (Chapman \& Hall/CRC, 2011).

${ }^{9}$ R. J. Seymour, M. R. Junnarkar, and R. R. Alfano, Phys. Rev. B 24, 3623 (1981).

10 J. M. Kikkawa and D. D. Awschalom, Phys. Rev. Lett. 80, 4313 (1998).

11 R. I. Dzhioev, K. V. Kavokin, V. L. Korenev, M. V. Lazarev, B. Ya. Meltser, M. N. Stepanova, B. P. Zakharchenya, D. Gammon, and D. S. Katzer, Phys. Rev. B 66, 245204 (2002).

12 J. H. Buß, J. Rudolph, F. Natali, F. Semond, and D. Häegele, Appl. Phys. Lett. 95, 192107 (2009).

13 M. Krauß, H. C. Schneider, R. Bratschitsch, Z. Chen, and S. T. Cundiff, Phys. Rev. B 81, 035213 (2010).

${ }^{14}$ K. Shen, Chin. Phys. Lett. 26, 067201 (2009).

15 J. H. Buß, J. Rudolph, S. Starosielec, A. Schaefer, F. Semond, Y. Cordier, A. D. Wieck, and D. Hägele, Phys. Rev. B 84, 153202 (2011).

16 H. Ma, Z. M. Jin, L. H. Wang, and G. H. Ma, J. Appl. Phys. 109, 023105 (2011).

17 M. W. Wu and C. Z. Ning, Phys. Stat. Sol. (b) 222, 523 (2000).

18 B. N. Murdin, K. Litvinenko, J. Allam, C. R. Pidgeon, M. Bird, K. Morrison, T. Zhang, S. K. Clowes, W. R. Branford, J. Harris, and L. F. Cohen, Phys. Rev. B 72, 085346 (2005).

19 S. Oertel, J. Hübner, and M. Oestreich, Appl. Phys. Lett. 93, 132112 (2008).

20 J. H. Jiang and M. W. Wu, Phys. Rev. B 79, 125206 (2009); 83, 239906(E) (2011).

${ }^{21}$ K. Zerrouati, F. Fabre, G. Bacquet, G. Bandet, J. Frandon, G. Lampel and D. Paget, Phys. Rev. B 371334 (1988).

22 P. E. Hohage, G. Bacher, D. Reuter, and A. D. Wieck, Appl. Phys. Lett. 89, 231101 (2006).

${ }^{23}$ K. L. Litvinenko, L. Nikzad, J. Allam, B. N. Murdin, C. R. Pidgeon, J. J. Harris, and L. F. Cohen, J. Supercon. 20, 461 (2007).

24 J. H. Buß, J. Rudolph, F. Natali, F. Semond, and D. Häegele, Phys. Rev. B 81, 155216 (2010).

25 Y. G. Zhu, L. F. Han, L. Chen, X. H. Zhang, and J. H. Zhao, Appl. Phys. Lett. 97, 262109 (2010).

${ }^{26}$ P. J. Rizo, A. Pugzlys, A. Slachter, S. Z. Denega, D. Reuter, A. D. Wieck, P. H. M. van Loosdrecht, and C.
H. van der Wal, New J. Phys. 12, 113040 (2010).

${ }^{27}$ K. L. Litvinenko, M. A. Leontiadou, Juerong Li, S. K. Clowes, M. T. Emeny, T. Ashley, C. R. Pidgeon, L. F. Cohen, and B. N. Murdin, Appl. Phys. Lett. 96, 111107 (2010).

28 E. A. Barry, A. A. Kiselev, and K. W. Kim, Appl. Phys. Lett. 82, 3686 (2003).

29 M. Römer, H. Bernien, G. Müller, D. Schuh, J. Hübner, and M. Oestreich, Phys. Rev. B 81, 075216 (2010).

30 G. A. Intronati, P. I. Tamborenea, D. Weinmann, and R. A. Jalabert, arXiv:1102.4753,

31 M. I. D'yakonov and V. I. Perel', Zh. Eksp. Teor. Fiz. 60, 1954 (1971) [Fiz. Tverd. Tela (Leningrad) 13, 3581 (1971)]; Sov. Phys. JETP 33, 1053 (1971) [Sov. Phys. Solid State 13, 3023 (1972)].

32 S. Kratzer and J. Frey, J. Appl. Phys 49, 4064 (1978).

33 M. Q. Weng, M. W. Wu, and L. Jiang, Phys. Rev. B 69, 245320 (2004).

34 M. Q. Weng and M. W. Wu, Phys. Rev. B 70, 195318 (2004).

35 P. Zhang, J. Zhou, and M. W. Wu, Phys. Rev. B 77, 235303 (2008).

36 J. Y. Fu, M. Q. Weng, and M. W. Wu, Physica E (Amsterdam) 40, 2890 (2008).

37 Y. Qi, Z. G. Yu, and M. E. Flatté, Phys. Rev. Lett. 96, 026602 (2006).

38 M. W. Wu and C. Z. Ning, Eur. Phys. J. B 18, 373 (2000); M. W. Wu, J. Phys. Soc. Jpn. 70, 2195 (2001).

39 M. W. Wu and H. Metiu, Phys. Rev. B 61, 2945 (2000).

40 I. Vurgaftman, J. R. Meyer, and L. R. Ram-Mohan, J. Appl. Phys. 89, 5815 (2001).

41 X. L. Lei, D. Y. Xing, M. Liu, C. S. Ting, and J. L. Birman, Phys. Rev. B 36, 9134 (1987).

42 N. Chand, T. Henderson, J. Klem, W. T. Masselink, R. Fischer, Y.-C. Chang, and H. Morkoç, Phys. Rev. B 30, 4481 (1984).

43 G. Dresselhaus, Phys. Rev. 100, 580 (1955).

44 E. L. Ivchenko and G. E. Pikus, Superlattices and Other Heterostructures: Symmetry and Optical Phenomena (Springer, Berlin, 1997).

45 T. Korn, Phys. Rep. 494, 415 (2010).

46 It is noted that Case (ii) is not presented because in the absence of the $L$ valleys, the multi-subband effect becomes important and the comparison between Case (ii) and the genuine case is meaningless.

47 H. Ma, Z. M. Jin, G. H. Ma, W. M. Liu, and S. H. Tang, Appl. Phys. Lett. 94, 241112 (2009).

48 J. H. Jiang and M. W. Wu, Appl. Phys. Lett. 96, 136101 (2010).

49 B. Y. Sun, P. Zhang, and M. W. Wu, J. Appl. Phys. 108, 093709 (2010).

50 B. Beschoten, E. Johnston-Halperin, D. K. Young, M. Poggio, J. E. Grimaldi, S. Keller, S. P. DenBaars, U. K. Mishra, E. L. Hu, and D. D. Awschalom, Phys. Rev. B 63, 121202(R) (2001).

51 J. Zhou, J. L. Cheng, and M. W. Wu, Phys. Rev. B 75, 045305 (2007).

52 W. J. H. Leyland, G. H. John, R. T. Harley, M. M. Glazov, E. L. Ivchenko, D. A. Ritchie, I. Farrer, A. J. Shields, and M. Henini, Phys. Rev. B 75, 165309 (2007).

53 X. Z. Ruan, H. H. Luo, Y. Ji, Z. Y. Xu and V. Umansky, 
Phys. Rev. B 77, 193307 (2008).

${ }^{54}$ L. F. Han, Y. G. Zhu, X. H. Zhang, P. H. Tan, H. Q. Ni and Z. C. Niu, Nanoscale Res. Lett. 6, 84 (2011).

55 M. M. Glazov and E. L. Ivchenko, Pis'ma Zh. Eksp. Teor. Fiz. 75, 476 (2002) [JETP Lett. 75, 403 (2002)]; Zh. Eksp. Teor. Fiz. 126, 1465 (2004) [JETP 99, 1279 (2004)].

56 M. A. Brand, A. Malinowski, O. Z. Karimov, P. A. Marsden, R. T. Harley, A. J. Shields, D. Sanvitto, D. A. Ritchie, and M. Y. Simmons, Phys. Rev. Lett. 89, 236601 (2002).

57 F. X. Bronold, A. Saxena, and D. L. Smith, Phys. Rev. B 70, 245210 (2004).

58 M. Q. Weng and M. W. Wu, Phys. Rev. B 68, 075312 (2003).

59 J. L. Birman, M. Lax, and R. Loudon, Phys. Rev. 145, 620 (1966).

${ }^{60}$ D. C. Herbert, J. Phys. C 6, 2788 (1973).

${ }^{61}$ W. Fawcett and D. C. Herbert, J. Phys. C 7, 1641 (1974).

${ }^{62}$ R. Mickevičius and A. Reklaitis, J. Phys.: Condens. Matter
2, 7883 (1990).

63 G. D. Mahan, Many-Particle Physics (Plenum, New York, 1990).

64 E. M. Conwell, High Field Transport in Semiconductors (Academic Press, New York, 1967).

65 X. L. Lei and C. S. Ting, Phys. Rev. B 30, 4809 (1984).

${ }^{66}$ K. Seeger, Semiconductor Physics: An Introduction (Springer, 2004).

67 X. L. Lei, Balance Equation Approach to Electron Transport in Semiconductors (World Scientific, Singapore, 2008).

68 Compared to the genuine condition, the drift velocity in the $L$ valleys stays almost unchanged by setting the electron-impurity scattering for $L$-valley electrons to zero but increases by more than $40 \%$ by removing the intervalley electron-phonon scattering for both $E=1$ and $6 \mathrm{kV} / \mathrm{cm}$ with $n_{e}=10^{18} \mathrm{~cm}^{-3}$ and $T=300 \mathrm{~K}$. 